\title{
Modeling diffusion and phase transitions by a uniform-acceptance force-bias Monte Carlo method
}

\author{
Maria Timonova, ${ }^{*}$ Jasper Groenewegen, and Barend J. Thijsse \\ Department of Materials Science and Engineering, Delft University of Technology, Mekelweg 2, 2628 CD Delft, The Netherlands
}

(Received 19 October 2009; revised manuscript received 1 March 2010; published 13 April 2010)

\begin{abstract}
The uniform-acceptance force-bias Monte Carlo (UFMC) method [G. Dereli, Mol. Simul. 8, 351 (1992)] is a little-used atomistic simulation method that has strong potential as alternative or complementary technique to molecular dynamics (MD). We have applied UFMC to surface diffusion, amorphization, melting, glass transition, and crystallization, mainly of silicon. The purpose is to study the potential and the limitations of the method: to investigate its applicability, determine safe and effective values of the two UFMC parameters-a temperature and a maximum allowed atomic displacement per iteration step - that lead to reliable results for different types of simulations, assess the computational speed increase relative to MD, discover the microscopic mechanisms that make UFMC work, and show in what kind of simulations it can be useful and preferable over MD. It is found that in many simulations, UFMC can be a very efficient alternative to MD: it leads to analogous results in much fewer iteration steps. Due to the straightforward formalism of UFMC, it can be easily implemented in any MD code. Thus both methods can be combined and applied in turn, using UFMC for the acceleration of certain processes and MD for keeping precision and monitoring individual atom trajectories.
\end{abstract}

DOI: $10.1103 /$ PhysRevB.81.144107

PACS number(s): $02.70 . \mathrm{Tt}, 02.70 . \mathrm{Ns}, 68.35$.Fx, 64.60.-i

\section{INTRODUCTION}

In materials science, true understanding begins at the atomic level. The concerted behavior of atoms determines the properties of a material and the effects of external influences to which the material is subjected. It has therefore been recognized long ago that molecular-dynamics (MD) simulation, with its atomic resolution and full dynamical evolution monitoring, is a powerful technique for materials modeling, especially since nonequilibrium conditions are often critical in the synthesis of materials. However, routes to equilibrium are equally important, and because the relaxation to equilibrium is often a time-consuming process in simulations, a method faster than MD is highly desirable.

A promising alternative to the MD is one of the Monte Carlo techniques based on the force-bias Monte Carlo (FBMC) method ${ }^{1}$ in which the probability of the transition of an atom from one position to the next is force dependent. Although FBMC has many advantages over other MC techniques and can be easily implemented into an MD simulation code, it has not been widely used. Only a few authors have applied it in simulations of water. ${ }^{2}$ Later the FBMC method was modified by Dereli ${ }^{3}$ by making the transition of an atom be always accepted. Dereli called it a continuum Monte Carlo method and applied it to study the growth of amorphous silicon. Another group has used this method for epitaxial growth of Ge on (100) Si (Ref. 4) and called it dynamic Monte Carlo. In both cases, the authors did not present values for all UFMC parameters and did not discuss the potential and limitations of the method. It was merely shown that these simulations gave physically meaningful results, comparable with $\mathrm{MD}$, but with the distinct advantage of a smaller number of calculation steps.

Encouraged by these results we decided to study the method more intensively and under more process conditions, to determine safe and effective values of the UFMC param- eters that lead to reliable results in different types of simulations, assess the computational speed increase relative to $\mathrm{MD}$, and identify the microscopic mechanisms that make UFMC work. The cases studied are an analytical onedimensional (1D) diffusion model, three-dimensional (3D) surface diffusion, a crystal at temperatures below melting, and phase transitions such as solidification, amorphization, recrystallization, and melting. The aim of this work is threefold. First, we want to explore how wide the applicability of the method is. A second objective is to determine what the method's main ways of operation are, in other words, which types of microscopic events are the actual carriers of the method and third, we want to find out by how much the computational speed can be accelerated compared to MD, without sacrificing too much physical fidelity. In the present paper, we name this method the uniform-acceptance forcebias Monte Carlo (UFMC) method to indicate that it is a particular case of FBMC.

The paper is organized as follows. It starts with a description of the UFMC method (Sec. II) and the computational details of the simulations (Sec. III). Thereafter, the simulation results are presented: the 1D idealized diffusion model and 3D $\mathrm{Cu}$ diffusion on a (100) $\mathrm{Cu}$ surface in Sec. IV A, a Si crystal below its melting point in Sec. IV B, phase transitions of $\mathrm{Si}$ in Sec. IV C, and recrystallization of an amorphous $\mathrm{Si}$ phase produced by ion bombardment on the (100) surface of a Si crystal in Sec. IV D.

\section{METHOD AND COMPUTATIONAL DETAILS}

\section{A. UFMC method}

During a UFMC simulation, atoms are moved iteratively like in MD. A major difference with MD is that in UFMC, the atomic velocities do not play a role, simply because they are not considered; only positions and forces count. In a 
single UFMC iteration step, all atoms are moved stochastically. Every atom is given probabilistic displacements $\delta_{v}(\nu=x, y, z)$ in the three Cartesian directions, limited by the values $-\Delta / 2$ and $\Delta / 2$, where $\Delta / 2$ is a user-selectable parameter of the method. Hence, $\Delta$ is the total range in which the new position of the atom will fall. Thus, $\delta_{v}$ normalized by $\Delta / 2$ or

$$
\xi_{v}=\frac{\delta_{v}}{\Delta / 2}
$$

takes values between -1 and +1 , and a uniform random number $\eta_{v}$ on $[0,1]$ is used to generate a value for $\xi_{v}$ in the following way:

$$
\xi_{v}=\frac{1}{\gamma_{v}} \ln \left[\eta_{v}\left(e^{\left|\gamma_{v}\right|}-e^{-\left|\gamma_{v}\right|}\right)+e^{-\left|\gamma_{v}\right|}\right],
$$

where

$$
\gamma_{v}=\frac{F_{v} \Delta / 2}{2 k_{B} T}
$$

with $F_{v}$ the $\nu$ component of the force $\mathbf{F}$ acting on the atom under consideration, $k_{B}$ the Boltzmann constant, and $T$ the temperature chosen for the UFMC simulation. The temperature $T$ is the second user-selectable parameter of the method. With Eq. (2), the probability density function of $\xi_{v}$ has the form

$$
p\left(\xi_{v}\right)=K_{v}^{-1} \exp \left(\frac{F_{v} \xi_{v} \Delta / 2}{2 k_{B} T}\right),
$$

where the constant $K_{v}$ is found by normalization on the defined interval $[-1,1]$. Then Eq. (4) becomes

$$
p\left(\xi_{v}\right)=\frac{\left|\gamma_{v}\right|}{\left(e^{\left|\gamma_{v}\right|}-e^{-\left|\gamma_{v}\right|}\right)} \exp \left(\gamma_{v} \xi_{v}\right) .
$$

For sufficiently small atomic moves, i.e., moves so small that the force does not change appreciably, this probability density function is equivalent to an exponential distribution of the change in the potential energy $U$ of the system as a result of the move. This is because the change in $U$ is given by $\delta_{U}=-F_{v} \delta_{v}$, which transforms the final exponential factor in Eq. (5) to

$$
\exp \left(\gamma_{v} \xi_{v}\right)=\exp \left(\gamma_{v} \frac{\delta_{v}}{\Delta / 2}\right)=\exp \left(-\frac{\delta_{U}}{2 k_{B} T}\right) .
$$

This is the essential condition of the UFMC method, and it shows-among other things-that atoms can make uphill moves, in the sense that a move can increase the potential energy. The factor 2 in the denominator of the exponent may seem strange in comparison to what one expects in a Boltzmann factor. However, it was reported by different authors ${ }^{2}$ that this factor yields a faster convergence most of the time. We will continue to refer to $T$ as "the" temperature in an UFMC simulation, although, as just mentioned, in UFMC the atoms have no velocity and therefore no kinetic energy.

Note that UFMC is not really a sampling method such as, for instance, Metropolis MC. In Metropolis MC, structural phase space is sampled according to a well-known equilib- rium ensemble distribution, for example, NVT (Gibbs petitcanonical ensemble), without any role of time as a driving quantity. Instead, UFMC approximates the dynamics of a system, irrespective of its distance from equilibrium. It is true, of course, that the atomic moves are sampled from a distribution [Eqs. (5) and (6)] but it is the entire dynamic route that is sampled, not just the final equilibrium distribution. Move-by-move, under UFMC each system change is driven by a Boltzmann distribution [Eq. (6)], which is different from the classical ensembles but may be seen as a long succession of instantaneous and local statistical processes. Our results will show that, if UFMC is applied inside its safe regime, the dynamic route and the final state are robust and stable.

From the user perspective, two parameters have to be chosen before applying the UFMC method: the temperature $T$ and the maximum allowed displacement $\Delta / 2$. In this work, we will show that, although $T$ is formally a parameter rather than a real temperature, realistic values should be chosen for $T$. For $\Delta / 2$, the selected values should be much smaller than a typical interatomic distance $R$, or else Eq. (6) will be severely violated or atoms will start approaching each other unphysically close. Yet, very small values for $\Delta / 2$ should be avoided because they will lead to an ineffective simulation and little or no gain in computational speed. Practical choices will be discussed in this work.

For convenience, we list a few analytical results. All expressions are based on the probability density of Eq. (5). As $\gamma_{v}$ is very often a number smaller than 1 , the small- $\gamma_{v}$ expressions are given separately. The average value of $\xi_{v}$ is

$$
\begin{gathered}
\left\langle\xi_{v}\right\rangle=\operatorname{coth} \gamma_{v}-\frac{1}{\gamma_{v}}, \\
\left\langle\xi_{v}\right\rangle=\frac{\gamma_{v}}{3}-\frac{\gamma_{v}^{3}}{45} \quad\left(\gamma_{v} \text { small }\right)
\end{gathered}
$$

with standard deviation

$$
\begin{gathered}
\sigma_{\xi}=\sqrt{1+\frac{1}{\gamma_{v}^{2}}-\operatorname{coth}^{2} \gamma_{v},} \\
\sigma_{\xi_{v}}=\sqrt{\frac{1}{3}}\left(1-\frac{\gamma_{v}^{2}}{10}\right) \quad\left(\gamma_{v} \text { small }\right) .
\end{gathered}
$$

The average change in the potential energy due to a single move in one direction is

$$
\left\langle\delta_{U}\right\rangle=-F_{v}\left\langle\delta_{v}\right\rangle=-F_{v}\left\langle\xi_{v}\right\rangle \Delta / 2 .
$$

The probability of an atom to move in the direction opposite to the force is

$$
\begin{gathered}
P\left(F_{v} \xi_{v}<0\right)=\frac{1-e^{-\left|\gamma_{v}\right|}}{e^{\left|\gamma_{v}\right|}-e^{-\left|\gamma_{v}\right|}}, \\
P\left(F_{v} \xi_{v}<0\right)=\frac{1}{2}-\frac{\left|\gamma_{v}\right|}{4} \quad\left(\gamma_{v} \text { small }\right) .
\end{gathered}
$$

In Fig. $1,\left\langle\xi_{v}\right\rangle, \sigma_{\xi}$, and $P\left(F_{v} \xi_{v}<0\right)$ are plotted as a function of $\gamma_{v}$ according to Eqs. (7), (9), and (12), respectively. It 


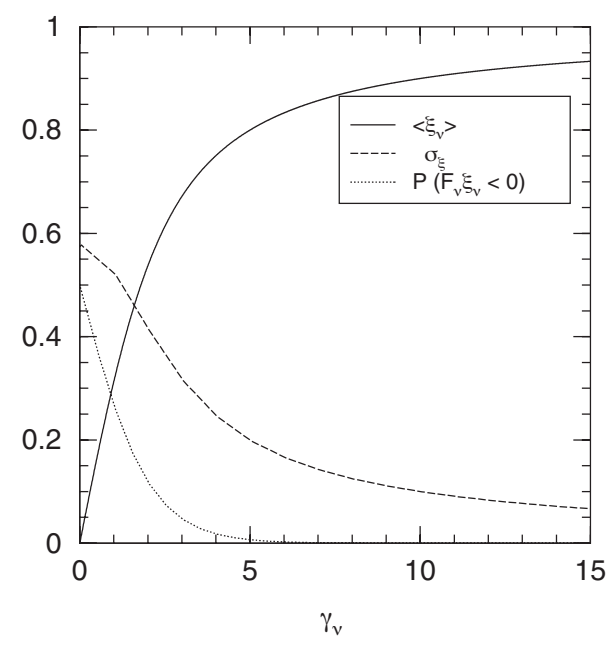

FIG. 1. Average value $\left\langle\xi_{v}\right\rangle$, standard deviation $\sigma_{\xi}$, and probability of an atom to move in the direction opposite to the force $P\left(F_{v} \xi_{v}<0\right)$ as a function of $\gamma_{v}$, according to Eqs. (7), (9), and (12), respectively.

is seen that $\left\langle\xi_{v}\right\rangle$ increases and $\sigma_{\xi}$ decreases with increasing $\gamma_{v}$. This means that if an atom experiences a large force (compared to $k_{B} T$ ), it moves on average over a large distance but the spread in this distance is small. A large force also reduces the probability of an uphill move, i.e., a move opposite to the force, which seems reasonable.

As illustration, Fig. 2 shows the full probability density function $p\left(\xi_{x}\right)$ of a particle move $\xi_{x}$ for three values of $\gamma_{x}$. The case shown is that for a particle in a one-dimensional quadratic potential well given by $E=0.25\left(\mathrm{eV} / \AA^{2}\right) x^{2}$, where $x$ is the location of the particle in the well, and for the choice $\Delta / 2=0.2 \AA$. The probability distribution is shown for $\gamma_{x}=1,0.5$, and 0.1 . At constant temperature, $T=580 \mathrm{~K}$, these three curves correspond to the cases where the particle posi-

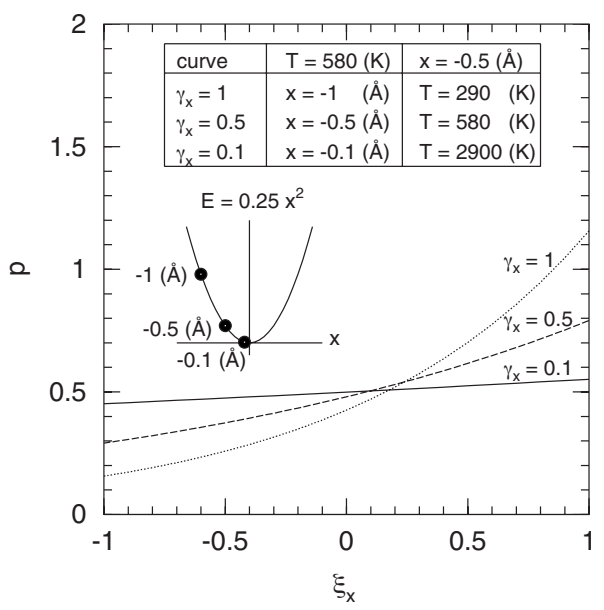

FIG. 2. Probability density function $p$ of the particle displacement $\xi_{v}$ in a one-dimensional quadratic potential well $E=0.25\left(\mathrm{eV} / \AA^{2}\right) x^{2}$, where $x$ is the position of the atom. Curves are plotted for $\gamma_{x}=1,0.5$, and 0.1 , referring either to the particle being at different locations $x=-1,-0.5$, and $-0.1(\AA)$ at $T=580(\mathrm{~K})$ or to different temperatures $T=290,580$, and $2900(\mathrm{~K})$ with the particle at $x=-0.5(\AA)$. In all cases, $\Delta / 2=0.2(\AA)$. tion before the move is $x=-1 \AA,-0.5 \AA$, and $-0.1 \AA$, respectively. Clearly, as the particle is further from equilibrium and experiences a larger force, the probability of the particle to move in the direction of the force is higher, as can be seen in the increasingly steeper slope of the curves. Alternatively, if one considers the initial particle position constant, $x=-0.5 \AA$, the three curves in Fig. 2 correspond to three temperatures, $T=290 \mathrm{~K}, 580 \mathrm{~K}$, and $2900 \mathrm{~K}$, respectively. From this viewpoint, one sees that lowering the temperature leads to an increasing probability of moves in the direction of the force. Thus by changing the parameter $T$, we can artificially increase or decrease the effect of the force on the displacement of the atom.

For completeness we also mention two extreme cases that may help understanding the UFMC. The first one is when $\gamma_{v}$ tends to zero, i.e., when the temperature $T$ is very high and/or the force $F_{v}$ on an atom is very small. In this case, the probability distribution becomes uniform, i.e., the move of the atom is purely random and independent of the force on it,

$$
\lim _{\gamma_{v} \rightarrow 0} \xi_{v}=\left(2 \eta_{v}-1\right)
$$

The other extreme case is the opposite: when the temperature $T$ is so low and/or the force $F_{v}$ on an atom is so large that $\gamma_{v}$ tends to infinity. Then the atom moves in the direction of the force over the maximum allowed distance,

$$
\lim _{\gamma_{v} \rightarrow \infty} \xi_{v}=\left\{\begin{array}{ll}
+1, & F_{v}>0 \\
-1, & F_{v}<0
\end{array} .\right.
$$

\section{B. UFMC simulation}

In UFMC simulations, in every iteration step, $3 N$ displacements (where $N$ is the number of atoms in the system) of the type of Eq. (2) are calculated, with the forces derived from the interatomic potential. As atomic velocities are not considered, time is no longer a sensible quantity. The duration of simulations is therefore measured in iteration steps. This makes it possible to compare the efficiency of MD and UFMC simulations since the extra time needed for UFMC [the evaluation of Eq. (2)] is generally negligible compared with the evaluation of the forces. It should be understood, of course, that the time step employed in MD was not given an unnecessarily small value to bias this efficiency difference. Also, with UFMC it is not possible to carry out simulations at constant pressure, as the pressure directly depends on the evaluation of the velocities (although one could choose to remove the kinetic term from the pressure tensor).

\section{UFMC + and UFMC ++ simulations}

In most cases, one wants to reestablish thermal equilibrium after a UFMC simulation, for example, because one wants to continue with MD simulation or simply in order to bring atoms closer to their equilibrium positions and reduce the unphysical variety in the potential energies. This has to start with attributing meaningful velocities to the atoms and let the system itself evolve to equilibrium. We have used two variants. One is giving zero velocities to all atoms and doing 
a short constant-temperature MD run with the thermostat set at $T$, the temperature used in the preceding UFMC simulation. The end product of this combined simulation, which we call $\mathrm{UMFC}+$, is a system in thermal equilibrium at temperature $T$. The second variant is, again, starting with zero atomic velocities but this time continuing with a constanttemperature $\mathrm{MD}$ run with the thermostat set at $0 \mathrm{~K}$. Of course the instantaneous temperature will momentarily become finite but the end product of this, so-called UFMC ++ simulation is a system quenched to $0 \mathrm{~K}$. In both cases, we find that $1.5 \mathrm{ps}$ is typically long enough for the MD run to reach equilibrium.

\section{SIMULATIONS}

\section{A. Simulations and motivation}

In order to systematically study the various possibilities, speed enhancements, and reliability limits of UFMC, and their dependence on the values of the parameters $T$ and $\Delta / 2$, we have performed a set of simulations starting from a simple model and ending with complex simulations of phase transitions and recrystalization of an amorphous phase. All results will be compared with MD simulations on the same systems. The results will be presented in order of increasing complexity:

(1) diffusion of a single particle in a $1 \mathrm{D}$ sinusoidal potential,

$$
U=\frac{Q_{0}}{2}\left[1-\cos \left(\frac{2 \pi x}{R}\right)\right],
$$

where $U$ is the potential energy of the system, consisting of one particle at position $x, Q_{0}$ is the potential barrier (or the activation energy), and $R$ is the period of the potential. This is a simple system, allowing easy access to all relevant information. We test UFMC by calculating, for different values of $\Delta / 2$, the number of jumps over the potential barriers per number of iteration steps, and by determining the apparent activation energy compared to $Q_{0}$ from runs at different $T$.

(2) 3D diffusion of a $\mathrm{Cu}$ adatom on a (100) $\mathrm{Cu}$ surface, also for different $T$ and $\Delta / 2$. This situation is closer to reality, yet it is still a single-atom process, i.e., there are no other processes disturbing the diffusion of the adatom. Again, our main objective is to find the apparent activation energies and the speed enhancements for various values of the UFMC parameters.

(3) A perfect Si crystal, periodic in all directions, kept at various constant temperatures below melting. The objective of these 3D bulk simulations is not to study atomic transition rates but to investigate how much UFMC will increase the energy, introduce disorder, and possibly create temporary vacancies that lead to diffusion, all in a system that is thermodynamically in a static state. Also we wanted to find out under which conditions the system will cease to return to its initial perfect-crystal state, thereby signaling the onset of unsafe simulation conditions. In short, these simulations were performed to explore the edges of UFMC. Somewhat moving up in complexity, we have also performed simulations of a Si crystal with two (100) surfaces, to see if the presence of a surface would possibly lead to the formation of unphysical surface features that could penetrate into the bulk.

(4) Formation of amorphous Si by cooling of the liquid and crystallization of the amorphous phase by heating. Using $\mathrm{MD}$, these are time-consuming simulations. Even worse, to produce an amorphous phase in a way compatible with the experiment, the cooling of the liquid should take microseconds or longer while MD allows atmost nanosecond-scale cooling simulations. In order to see what time acceleration can be reached with UFMC and how reliable the results are, we have performed simulations of phase transitions by UFMC+ and compared it with MD.

(5) Recrystallization of an amorphous phase formed on top of a $\mathrm{Si}$ crystal formed by bombardment of a (100) $\mathrm{Si}$ surface by $500 \mathrm{eV}$ Ar atoms. Again, in MD these are timeconsuming simulations, and UFMC can be used to speed them up. However, recrystallization is a complex process, and we wanted to investigate if all aspects of the process, or just the recystallized end product, will be similar in MD and UFMC, and how close this similarity is. For this, UFMC ++ simulations of annealing the amorphous/crystal system were performed at different temperatures $T$ and with different $\Delta / 2$ values.

\section{B. Simulation details}

The UFMC and MD simulations of copper (001) adatom diffusion were performed for a crystal of $25.3 \AA$ thickness with a surface area of $32.5 \times 32.5 \AA^{2}$ or $9 \times 9$ unit cells. The $\mathrm{Cu}-\mathrm{Cu}$ interactions were described by the Oh-Johnson embedded atom method (EAM) potential. ${ }^{5}$

The UFMC and MD simulations of pure silicon with the cubic diamond structure were performed for a $81 \times 81 \times 81 \AA^{3}$ system, containing $27000 \mathrm{Si}$ atoms. This density corresponds with the experimental value at $0 \mathrm{~K}$. Si-Si interactions were described with a recent parameterization of the modified embedded atom method potential, called MEAM-L. With the exception of the too high melting temperature $(2990 \mathrm{~K})$, this potential describes silicon properties better that many other potentials., ${ }^{6,7}$ All UFMC and MD simulations were done at constant volume unless (for MD) indicated otherwise.

The UFMC+ and MD simulations of phase transitions during cooling liquid $\mathrm{Si}$ and heating amorphous $\mathrm{Si}$ were done for a system of 13824 atoms. The MD simulation was performed at constant atmospheric pressure. Si liquid was cooled to room temperature and subsequently heated to the melting temperature with a cooling/heating rate of $0.91 \mathrm{~K} / \mathrm{ps}$. In the UFMC simulation, the initial liquid and amorphous phases were obtained from MD. Therefore, the volumes of the liquid and amorphous phase were different, $279.2 \mathrm{~nm}^{3}$ and $273.6 \mathrm{~nm}^{3}$, respectively. This difference between UFMC and MD is not considered essential, as a volume change in this size makes only a very minor contribution to the potential energy compared with the phase transitions themselves.

In the UFMC ++ and MD annealing simulations of the amorphous phase formed by $500 \mathrm{eV}$ Ar sputter bombardment of a Si crystal, the amorphous phase was $20 \AA$ thick and contained Ar impurities. The system, with a surface area of 


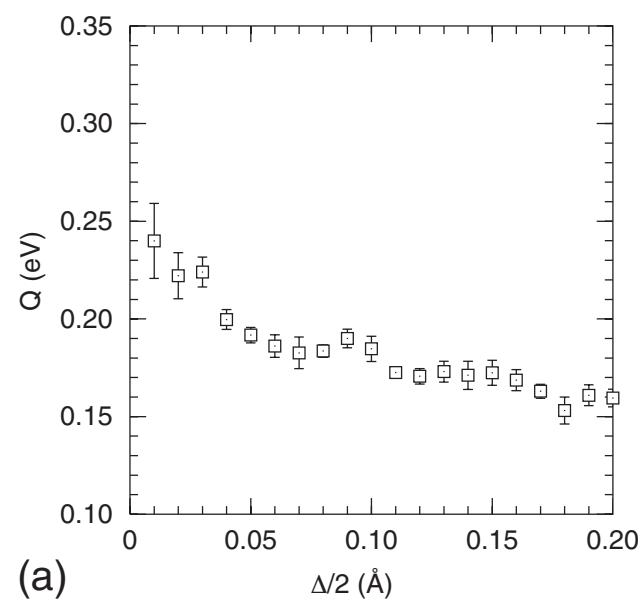

$81 \times 81 \AA^{2}$, consisted of $195 \mathrm{Ar}$ and $25209 \mathrm{Si}$ atoms. The MD simulations of the Ar bombardment and the subsequent annealing at different temperatures will be reported later. ${ }^{8}$ Ar-Si and Ar-Ar interactions were described with the FirsovMolière pair potential. ${ }^{9}$ This potential was also used for $\mathrm{Si}-\mathrm{Si}$ interactions at short distances.

In general, MD does not use a maximum displacement parameter in the same way as UFMC. However, the current MD implementation does employ a distance parameter $r_{\mathrm{m}}$. It has the following role. In each iteration, the MD code scales the time step in such a way that no atom in the system will travel a distance greater than $r_{\mathrm{m}}$ given its velocity and acceleration. The value for $r_{\mathrm{m}}$ normally used in MD is $0.02 \AA$, as followed from numerical experiments of computational efficiency versus dynamical precision, and this distance could be regarded as a maximum displacement distance in the same spirit as $\Delta / 2$. It is much smaller than the values that we will use for $\Delta / 2$. In order to examine whether an increase in $r_{\mathrm{m}}$ would speed up the simulations in the same way as UFMC, we have also performed MD simulations with a higher $r_{\mathrm{m}}$ value, $0.14 \AA$ (Secs. IV A and IV B). All MD simulations were run with the velocity Verlet integration method, employing the maximum-distance method just mentioned for automatic time step determination. As an example, for the system at room temperature, the average time step is $1.2 \mathrm{fs}$, and at $2024 \mathrm{~K}$ it has dropped to $0.5 \mathrm{fs}$. The overall temperature of the system was controlled by using Berendsen damped velocity scaling with a time constant of $18 \mathrm{fs}$, applied to all atoms in the system.

\section{Quasivacancies}

To be able to identify pointlike defects that can be defined in atomic models of different kinds of condensed matter, we make use of the concept of quasivacancies (QVs). These are defined as follows. An atom $i$, at position $\mathbf{r}_{i}$, is said to have an associated "missing atom" if the vector $\Delta \mathbf{R}_{i} \equiv \Sigma_{j}\left(\mathbf{r}_{j}-\mathbf{r}_{i}\right)$, where the atoms $j$ are the neighbors of $i$, has a length of at least $80 \%$ of the nearest-neighbor distance (using $80 \%$ instead of $100 \%$ allows for some structural and thermal disorder). The missing atom is then considered to lie at $\mathbf{r}_{\mathrm{MA}}=\mathbf{r}_{i}-\Delta \mathbf{R}_{i}$. We next scale the missing atom concentration into a $\mathrm{QV}$ concentration by dividing it by the local coordi- nation number $Z$ of the atoms (in this case, $Z=4$ everywhere in the system). This yields a defect concentration that, in a crystal, exactly matches the true vacancy concentration. Hence the name quasivacancies. The advantage of quasivacancies is that they are defined not only in a crystal but also in a liquid or amorphous phase.

\section{RESULTS}

\section{A. Diffusion}

\section{One-dimensional diffusion}

1D simulations of the diffusion of a particle in the simple sinusoidal potential of Eq. (16) were performed for a barrier energy $Q_{0}=0.25 \mathrm{eV}$ and a period length $R=1 \AA$. Values up to $0.5 \AA$ were used for $\Delta / 2$, which is $50 \%$ of $R$, i.e., $50 \%$ of the jump distance. Values between 500 and $850 \mathrm{~K}$ were used for $T$. For each $\Delta / 2$, the apparent activation energy was found by fitting the logarithm of the number of jumps versus $1 / T$ by the Arrhenius expression,

$$
\ln \left(\frac{n}{N_{I}}\right)=-\frac{Q}{k_{B} T}+\ln \left(\frac{\nu_{0} t}{N_{I}}\right),
$$

where $n$ is the number of jumps observed in $N_{I}$ iteration steps and $Q$ is the apparent activation energy. The attempt frequency $\nu_{0}$ and time duration $t$ are quantities that have no meaning in UFMC itself but spring into existence as soon as the Arrhenius picture is joined in. The apparent activation energy $Q$ is shown as a function of $\Delta / 2$ in Fig. 3(a). Each energy is calculated as the mean slope resulting from the 20 weighted least-square fits of the $\ln \left(n / N_{I}\right)$ data as a linear function of $1 / T$ [Eq. (17)] for each of 20 replica runs. The error bar shows the rms error of this average slope. It is found that $Q$ is virtually equal to $Q_{0}$ for very small $\Delta / 2$ and decays to $0.16 \mathrm{eV}$, or $0.65 \times Q_{0}$, for $\Delta / 2=0.20 \AA$. This means that increasing $\Delta / 2$ to $20 \%$ of the jump distance leads to a significant decrease in the energy barrier, and therefore to an easier activation of particle diffusion. Clearly this decrease in the energy barrier is one of the mechanisms by which UFMC operates.

The fact that for small values of $\Delta / 2$, the apparent activation energy is very nearly equal to $Q_{0}$ shows that the UFMC parameter $T$ can be considered as a realistic tempera- 


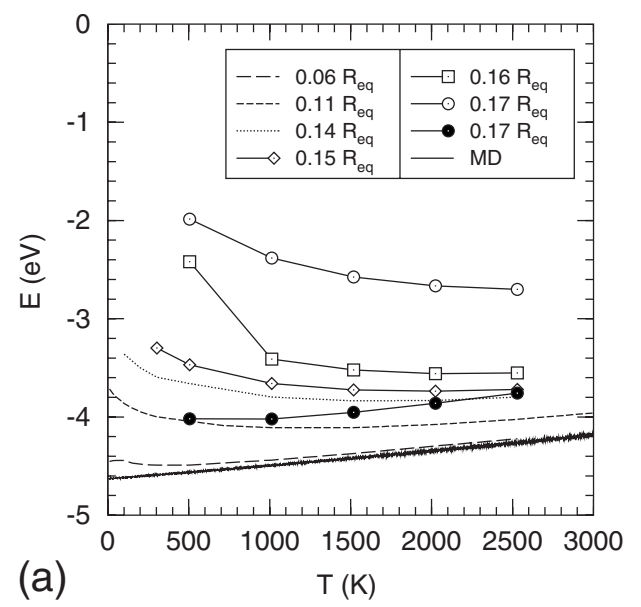

ture, and that the use of the factor 2 in Eqs. (3) and (6) is therefore justified. To see this, one should realize that if we would have used a UFMC version without the seemingly suspicious factor 2 in Eq. (4) or (6), we would have obtained from the fit of Eq. (17) a $Q$ that would be exactly two times as large as the $Q$ that we have found here. This would have led to a very unrealistic Fig. 3(a). Later we will find more evidence of the functioning of $T$ as a realistic temperature.

Although these results are complete by themselves, from the intercepts of the fits of Eq. (17) the simulated time per iteration step $t / N_{I}$ can be estimated as a function of $\Delta / 2$ for a chosen practical case. For this, we use $\nu_{0}=1.6 \times 10^{13} \mathrm{~s}^{-1}$, a value calculated directly from transition state theory ${ }^{10}$ and a harmonic potential for copper. We find that $t / N_{I}$ increases with $\Delta / 2$, reaching the value $1.7 \mathrm{fs} / \mathrm{step}$ for $\Delta / 2=0.20 \AA$. Again it should be mentioned that in order to make the translation from UFMC iteration steps to time, one needs an independent time-related quantity as additional input.

\section{Three-dimensional simulation of surface diffusion}

In three-dimensional UFMC and MD simulations of surface diffusion of a $\mathrm{Cu}$ adatom on a fcc (001) $\mathrm{Cu}$ surface, the temperature range from 500 to $1050 \mathrm{~K}$ was explored. The UFMC simulations were performed with $\Delta / 2=0.27 \AA$. This value is $11 \%$ of the distance between nearest adatom positions, which is the same as the nearest-neighbor distance $R_{\text {eq }}=2.56 \AA$. The relative number of jumps of the $\mathrm{Cu}$ adatom, $n / N_{I}$, versus the inverse of the temperature is shown in Fig. 3(b). From the fit of Eq. (17), the activation energy $Q$ was found to be $0.26 \mathrm{eV}$ for UFMC and $0.39 \mathrm{eV}$ for MD, both with an uncertainty of $10 \%$. The MD value of the activation energy is in excellent agreement with the experiment, $0.4 \mathrm{eV} .^{11}$ The UFMC value is about $67 \%$ of the MD value. This is not very far from the approximate ratio 0.7 that is seen in Fig. 3(a) for $\Delta / 2=0.11 \AA$. It suggests that the percentage lowering of the activation energy may be a universal function of $(\Delta / 2) / R$, where $R$ is the jump distance. More work is needed in this area.

From the intercept of the fit of Eq. (17) to the UFMC results, the simulated time per iteration step $t / N_{I}$ was calculated using $\nu_{0}=1.6 \times 10^{13} \mathrm{~s}^{-1}$ found from the MD simulation. The resulting $t / N_{I}$ value is $1.7 \mathrm{fs} / \mathrm{step}$, compared to the MD value $0.9 \mathrm{fs} / \mathrm{step}$, a speed boost is of a factor 1.9.
FIG. 4. Temperature dependence of the average atomic potential energy $E$ in crystalline silicon modeled by $\mathrm{MD}$ and by UFMC using different $\Delta / 2$. The filled circles are UFMC + results for $\Delta / 2=0.17 R_{\text {eq }}$.
Increase in the MD parameter $r_{\mathrm{m}}$ also accelerates simulations. As an example, an MD simulation of the adatom diffusion executed with $r_{\mathrm{m}}=0.14 \AA$, seven times larger than the normal value $0.02 \AA$, runs $67 \%$ faster for the same number of observed atom jumps. Therefore, for this type of simulation result, the MD method also offers a viable speed increase method. However, as we will see later, in other cases UFMC will be preferable.

\section{B. Si crystal}

UFMC simulations of diamond-cubic $\mathrm{Si}$ were done at different temperatures $T$ in the range from 10 to $2500 \mathrm{~K}$, which is below the melting point $2990 \mathrm{~K}$ according to the MEAM-L potential, and using values for $\Delta / 2$ between 0.134 and $0.403 \AA$ or $6-17 \%$ of the nearest-neighbor distance $R_{\text {eq }}=2.35 \AA$. In relative sense, this $\Delta / 2$ range is comparable to the range used for the 1D diffusion [Fig. 3(a)]. For comparison it is noted that the rms vibrational displacement of the $\mathrm{Si}$ atoms is $0.134 \AA$ at $T=170 \mathrm{~K}$ and $0.403 \AA$ at $T=1830 \mathrm{~K}$ according to MD. It may be tempting to use these values as an approximate way to map $\Delta / 2$ length values onto thermal disorder values but see below. As UFMC does not use classical mechanics to calculate the trajectories (moves) of atoms, contrary to MD, we perform this study to analyze the differences between UFMC and MD in general terms and from different viewpoints. As there are no vacancies in the system, we should not expect diffusion to take place in the crystal, at least not at first; these simulations were therefore not intended to study the lowering of an effective activation energy. Also, because no significant events happen in the MD simulations (apart from atomic vibrations), there is no gain in computational time that could be defined. Rather, we seek to find out how UFMC influences a perfect crystal in terms of its structure, energy, and phase transitions. The graphs shown display average values after the simulations have reached their steady state.

Let us first look at the distribution of the potential energies of the atoms in the crystal. Figures 4(a) and 4(b) show the average value $E$ and the standard deviation $\sigma_{E}$ as a function of $T$ for UFMC and MD. Clearly UFMC produces extra potential energy compared to $\mathrm{MD}$, the extra amount increasing rapidly with $\Delta / 2$ but decreasing with $T$. The same trend 


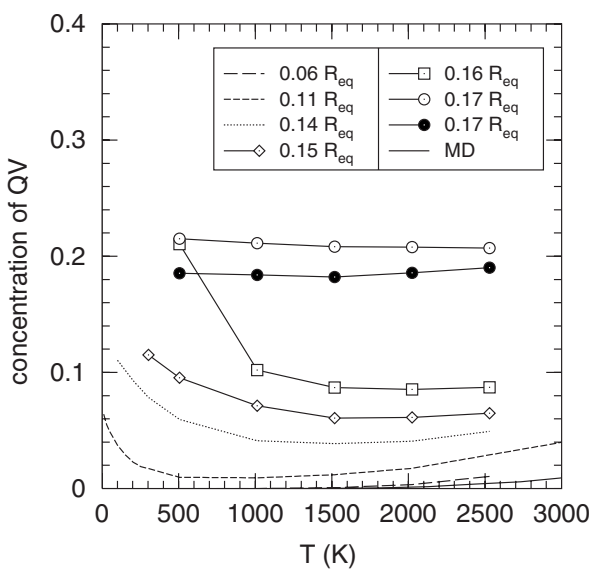

FIG. 5. Temperature dependence of the quasivacancy concentration in crystalline silicon modeled by MD and by UFMC using different $\Delta / 2$. The filled circles are UFMC+ results for $\Delta / 2=0.17 R_{\text {eq }}$.

is found for $\sigma_{E}$. We can understand this by realizing that, atomic forces being equal, the quantity $\gamma_{v}$ also increases with $\Delta / 2$ and decreases with $T$, see Eq. (3). Figure 1 shows that this, in turn, causes the average magnitude of the atomic moves, $\left\langle\xi_{v}\right\rangle \Delta / 2$, to increase with $\Delta / 2$ and decrease with $T$. Consequently, we can understand that larger average UFMC moves lead to more energy and more energy spread in the system. This is a very reasonable result. It should be noted, however, that the values of the energy increase and the energy spread increase by UFMC are quite considerable, as Figs. 4(a) and 4(b) show. The jump in the results for $\Delta / 2=0.16 R_{\text {eq }}$ when the temperature decreases from 1000 to $500 \mathrm{~K}$ turns out to be a universal feature in these simulations. We will find it later, too, and discuss it there.

To find out to what extent the difference in $E$ is related to the formation of defects, we will use the temperature dependence of the concentration of QVs, defined in Sec. III C. Figure 5 displays the crystal-averaged QV concentrations as a function of $T$ for different $\Delta / 2$. The curves show the same trends as the mean potential energy in Fig. 4(a): the differences with MD increase with $\Delta / 2$ and decreases with $T$. Also the jump in the results for $\Delta / 2=0.16 R_{\text {eq }}$ between 500 and $1000 \mathrm{~K}$ is seen in these data. Note that the concentration of quasivacancies in UFMC can become very high while in the MD simulation even near the melting point, the concentration stays below $1 \%$. It should be noted that in terms of QV concentrations there is a great difference between UFMC and $r_{\mathrm{m}}$-increased $\mathrm{MD}$ as speed enhancement methods. If $r_{\mathrm{m}}$ is increased by a factor of 8 , or even 15 , which makes it equal to $7 \%$ or $13 \%$ of $R_{\text {eq }}$, the QV concentration in the MD simulation hardly changes, and it certainly does not become as high as in UFMC with $0.06 R_{\text {eq }}$ or $0.14 R_{\text {eq }}$, respectively.

Two questions come up in relation to the energy increase and the defect production by UFMC. One is whether or not the system will settle down to the crystalline state if the UFMC simulation is extended by MD (i.e., if a UFMC+ simulation is performed). The other question is even more stringent, namely, whether after UFMC ++ each atom has resumed its original crystal position.

UFMC+ simulations were performed to answer the first question. It turns out that there is a crucial difference be- tween the results for $\Delta / 2=0.17 R_{\text {eq }}$ and those for lower $\Delta / 2$. For lower $\Delta / 2$, the MD part of UFMC+ returns the energy $E$, $\sigma_{E}$, and the quasivacancy concentration of the system to the proper MD values, i.e., to the values as if no UFMC had occurred. In other words, for lower $\Delta / 2$, the diamond-cubic $\mathrm{Si}$ crystal is recovered. In contrast, for $\Delta / 2=0.17 R_{\text {eq }}$, and most probably also for higher values, MD cannot return the system to the crystal phase after UFMC and leaves it in a high-energy, high-defect state [full circles in Figs. 4(a), 4(b), and 5]. In fact, as we will shortly see (Fig. 9), the structure is not far from the liquid or amorphous structure. One may therefore conclude that for UFMC applied to this particular system there is a sharp "phase boundary" between $\Delta / 2=0.16 R_{\text {eq }}$ and $0.17 R_{\text {eq }}$. As an interesting exception, the $500 \mathrm{~K}$ UFMC results for $0.16 R_{\mathrm{eq}}$ should be grouped with those for $0.17 R_{\text {eq }}$; this system, too, stays in the disordered state after MD. Apparently this was the significance of the jump in the results for $\Delta / 2=0.16 R_{\text {eq }}$ between 500 and $1000 \mathrm{~K}$ mentioned earlier. Again these results show that too high values of $\Delta / 2$, especially at low $T$, may be unsafe.

To answer the second question, whether or not atoms return to their original crystal position, we performed a set of UFMC ++ simulations and calculated for each atom the quantity $\Delta n_{\text {da }}$, being the number of departed "old" neighbors plus arrived "new" neighbors, comparing the situations before and after UFMC ++. A single swap of two atoms in the crystal is accompanied by $\Delta n_{\mathrm{da}} \geq 6$ neighbors (at least three neighbors depart and three others arrive) for each of the atoms. Therefore, we consider $\Delta n_{\mathrm{da}}=6$ as a minimal indication of an atomic jump. It is found that after UFMC ++ with $\Delta / 2 \leq 0.11 R_{\text {eq }}$, all the atoms are placed at the original positions for all UFMC temperatures, showing that the QVs formed during the UFMC phase appear as parts of temporary Frenkel pairs. In contrast, with $\Delta / 2=0.14 R_{\text {eq }}$, at $T=101 \mathrm{~K}$, $4.1 \%$ of atoms have $\Delta n_{\mathrm{da}} \geq 6$ after $10^{4}$ iteration steps. Already at $202 \mathrm{~K}$, this fraction decreases to $0.04 \%$ and it becomes even smaller with increasing $T$. A zero value is observed at $1012 \mathrm{~K}$ and at $2024 \mathrm{~K}$, the number has slightly increased to $0.002 \%$. This trend is very similar to the trend of the average atomic potential energy. We conclude that when the average potential energy is high (at low $T$ ), the conditions are favorable for the exchange of atomic positions. We do not call this process diffusion, as in this perfect crystal there is no diffusion at low temperatures. We will use the term "exchanges of atomic positions" because the percentage of atoms with $\Delta n_{\mathrm{da}}>8$ (these atoms certainly form point defects) at $101 \mathrm{~K}$ is negligible (only $0.02 \%$ ) and rapidly decreases to zero with $T$.

At this point, we want to make the following remark. The fact that there are parameter ranges for which UFMC ++ simulations leave a perfect crystal fully intact does not mean that within those parameter ranges UFMC ++ cannot accomplish anything. On the contrary, it only shows that processes such as vacancy-interstitial pair creation, with very high activation energies, do not occur spontaneously under UFMC ++ (in the time regime of the simulation). All processes with smaller activation energies, however, will certainly have a finite probability of occurring under $\mathrm{UFMC}++$, the probability increasing as the activation energy gets smaller and this is also what we observe. 
We will next investigate whether the average atomic potential energy $E$ during UFMC is a good indicator of atomic position exchange in the crystal after UFMC + or UFMC ++ . First of all, for $\Delta / 2=0.14 R_{\text {eq }}$, with increasing $T$ the percentage of atomic exchanges becomes negligibly small already at $202 \mathrm{~K}$, when $E$ is approximately $-3.5 \mathrm{eV}$. Therefore, we may consider this value as the lower-energy limit. It is supported by the fact that for $\Delta / 2 \leq 0.11 R_{\text {eq }}$, at all $T$, when the potential energy never exceeds $-3.5 \mathrm{eV}$, no single atomic swap is observed, and for $0.15 R_{\text {eq }}$, at $303 \mathrm{~K}$, when $E=-3.3 \mathrm{eV}$, already $4.6 \%$ of the atoms have changed at least six neighbors. At higher $T$, the potential energy decreases and simultaneously the percentage decreases to nearly zero. Also, for $\Delta / 2 \geq 0.16 R_{\text {eq }}$, when $T$ is above $1000 \mathrm{~K}$ and $E=-3.5 \pm 0.05 \mathrm{eV}$, the system returns to the crystal phase after UFMC+. All evidence points to $-3.5 \mathrm{eV}$ as the lower-energy limit for atomic exchange. An alternative formulation is $1.1 \mathrm{eV}$ above the ground-state $(0 \mathrm{~K})$ energy of the perfect crystal.

In order to find out if a surface can act as a nucleation point for the disordering of the type that was observed without surfaces for $\Delta / 2=0.17 R_{\text {eq }}$, UFMC was applied with smaller values, $\Delta / 2 \leq 0.14 R_{\text {eq }}$, to a $\mathrm{Si}$ crystal with a free and a fixed (100) surface. Atoms at the fixed surface were harmonically bound to their crystal locations so that nothing significant was expected to happen for these atoms. If any defect nucleation would occur, it would happen at the free surface. However, no such thing was observed. In fact, the energies during UFMC with surfaces were very similar to the ones shown in Fig. 4(a) without surfaces. In addition, after a small number of iteration steps, the free surface dimerized. All this is reassuring and indicates that UFMC may be applied equally well in the bulk as at a surface.

In conclusion, a careful choice of the parameters $T$ and especially $\Delta / 2$ is needed to prevent UFMC + simulations from driving a system so far from its state that it will no longer return to equilibrium. For the present case, crystalline silicon, we find that $\Delta / 2=0.16 R_{\text {eq }}$ is the highest value that can be applied just safely, although at low temperatures (below $1000 \mathrm{~K}$ ), this value is already too high, and 0.15 or 0.14 will be safer. Even so, the fact that the system returns to its equilibrium state does not mean that all the atoms at their original positions. If the atomic potential energy reaches a value above $-3.5 \mathrm{eV}$ during UFMC, which is more probable at lower $T$, atoms tend to swap. If the system consists of a single element, this may not cause any trouble. But in a system of two or more elements, swaps of atoms may cause formation of undesirable defects that prevent the system from returning to the original crystal phase after UFMC+ or $\mathrm{UFMC}++$. In this case, the value of potential-energy limit should be recalculated according to the specific elements and equilibrium structure. In general, the results appear to show that creation of quasivacancies and atomic swaps very likely is the essential mechanism by which UFMC reaches equilibrium, and that this may lead to large computational accelerations when the concentration of these defects is sufficiently high. As mentioned earlier, the same acceleration cannot be reached by increasing the MD parameter $r_{\mathrm{m}}$, as this does not lead to the formation of quasivacancies. We will have a further look at these advantages of UFMC over MD in the next section. Clearly if exchange of atomic positions cannot be tolerated at all in a particular application, UFMC should only be used in conditions that are absolutely safe. Note that we claim no universality of the present results. For example, some of the observations may be quite different for a closepacked crystal such as $\mathrm{Cu}$.

\section{Phase transitions}

To find out if the UFMC method can be applied to study phase transitions and what values of $\Delta / 2$ should be used, we have performed UFMC+ simulations of cooling liquid Si to room temperature and heating amorphous Si from room temperature to the melting point $(2990 \mathrm{~K})$. The simulations were done with $\Delta / 2=0.06 R_{\mathrm{eq}}, 0.11 R_{\mathrm{eq}}$, and $0.14 R_{\mathrm{eq}}$, at temperatures $T$ from 303 to $3036 \mathrm{~K}$. During cooling and heating, the temperature was changed in jumps of either 200 or $300 \mathrm{~K}$, and at each new temperature, $2 \times 10^{5}$ UFMC iteration steps were executed. This makes the average cooling/heating rate $1 \mathrm{mK} / \mathrm{step}$ or $1.5 \mathrm{mK} / \mathrm{step}$, respectively. At the end of every $2 \times 10^{5}$ steps, the configurations were saved and brought to real equilibrium in separate MD runs (or rather: real metastable equilibrium).

The initial amorphous and liquid systems for the UFMC+ simulations were taken from MD simulations of cooling the liquid and reheating it to the melting point with a cooling/heating rate of $0.91 \mathrm{~K} / \mathrm{ps}$ (Ref. 7). For comparison with the UFMC+ simulations, the potential energy during these cooling and heating $\mathrm{MD}$ runs is shown in Figs. 6(a)-6(c), along with that during a third MD run of heating crystalline Si. The MD results show amorphization (A) of the liquid around $2000 \mathrm{~K}$ during cooling and a glass transition $(\mathrm{G})$ at $2100 \mathrm{~K}$ during subsequent heating, followed by crystallization (C) at $2350 \mathrm{~K}$ and melting $(\mathrm{M})$ at $3050 \mathrm{~K}$. Here all temperatures are quoted at the halfway points of the transitions. The crystallized MD system was polycrystalline with a biggest grain of about $45 \mathrm{~nm}^{3}$ in the whole sample of $273.6 \mathrm{~nm}^{3}$.

It is seen in Fig. 6(a) that UFMC+ with $\Delta / 2=0.06 R_{\text {eq }}$ very closely reproduces the MD results of cooling the liquid. Again this can be seen as an indication that the temperature parameter employed in UFMC is in fact a realistic temperature. At room temperature, the amorphous phase is well relaxed and the potential energy matches the MD value within $0.005 \mathrm{eV} /$ atom. Here, the reduced radial distribution function (RDF) is used to characterize a liquid or amorphous phase. It is defined as

$$
G(r)=4 \pi r\left[d(r)-d_{0}\right]
$$

with $d(r)$ the average number density at a distance $r$ from an atom in the system and $d_{0}$ the overall number density in the system. The RDFs for the amorphous phases at $303 \mathrm{~K}$ simulated by UFMC+ and MD are presented in Fig. 7. They are very much alike, with the average coordination number for UFMC+ (4.25) being slightly lower than for MD (4.61). This difference in coordination number is accompanied by a slightly higher concentration of QVs (17\%) compared to that in $\mathrm{MD}(7.4 \%)$. Interestingly, the small secondary peak in the MD curve at $3 \AA$ is absent for UFMC+. The reason for this 

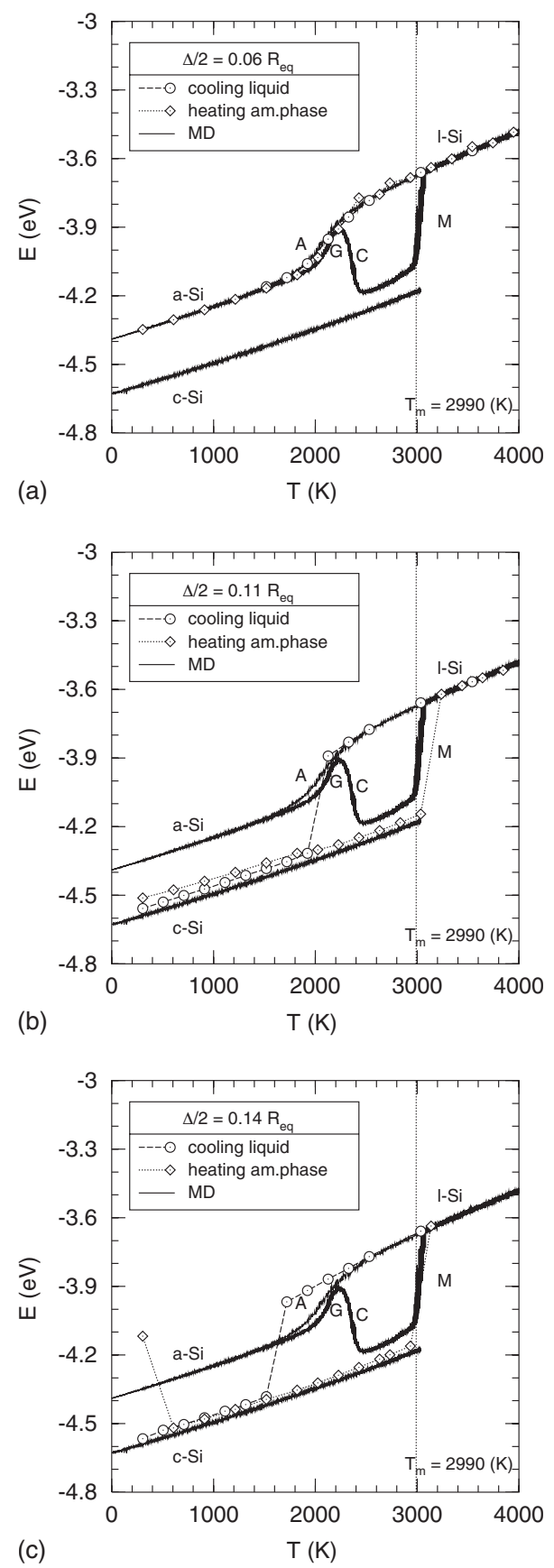

FIG. 6. Average atomic potential energy of silicon during heating the crystal, cooling the liquid, and heating the amorphous phase. The continuous lines show MD results for heating/cooling rates of $0.91(\mathrm{~K} / \mathrm{ps})$ taken from Ref. 7, the data points show UFMC+ results with $\Delta / 2=$ (a) $0.06 R_{\text {eq }}$, (b) $0.11 R_{\text {eq }}$, and (c) $0.14 R_{\text {eq. }}$ (A) Amorphization of the liquid, $(\mathrm{G})$ glass transition, $(\mathrm{C})$ crystallization of the amorphous phase, and (M) melting of the crystallized system.

difference is unknown. On heating, the amorphous phase does not crystallize as in MD. In a sense, this situation is similar to MD with a ten times higher heating rate $(9.1 \mathrm{~K} / \mathrm{ps})$ than used here, for which also no crystallization was observed. ${ }^{7}$ There seem to be simply not enough mobility for crystallization. Apparently the small value $\Delta / 2=0.06 R_{\text {eq }}$ prevents crystallization by holding atoms too closely near their positions in the amorphous phase, and the other UFMC

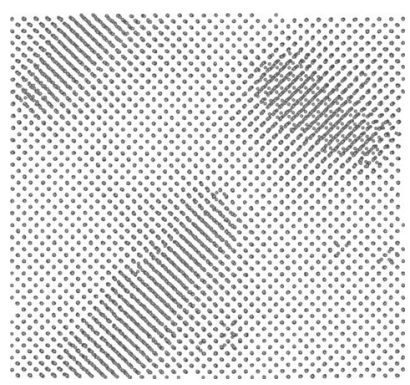

(a)

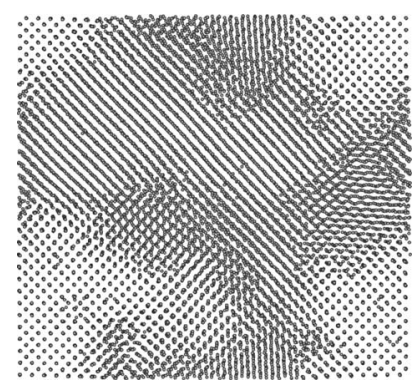

(b)
FIG. 7. Side views of polycrystalline silicon at 303 (K) formed in UFMC+ simulations with $\Delta / 2=0.11 R_{\text {eq }}$. during (a) cooling the liquid and (b) heating the amorphous phase.

parameter $T$ cannot change this, even while at low- $T$ atoms move over longer distances and while at high- $T$ atoms move more often in the direction opposite to the force and could be expected to cross the energy barrier between the amorphous and crystal phases. It should be stressed that this absence of crystallization is observed for a homogeneous system, one without nuclei for crystallization.

We conclude that with the small value $\Delta / 2=0.06 R_{\text {eq }}$, UFMC + can be used to form a well-relaxed amorphous phase from the liquid but not to crystallize the amorphous phase. The acceleration that can be obtained by using UFMC + is very high. It takes at least two orders of magnitude fewer iteration steps to produce the UFMC+ cooling curves than the MD cooling curves in Fig. 6(a), and both lead to virtually the same amorphous state.

A different behavior is observed during UFMC+ with larger $\Delta / 2$. For $\Delta / 2=0.11 R_{\text {eq }}$, we see in Fig. 6(b) that instead of amorphization of the liquid during cooling, the system crystallizes at $2000 \mathrm{~K}$. Also, instead of a gradual liquefaction of the amorphous phase during heating, the system crystallizes, already at $300 \mathrm{~K}$. The melting of these crystals occurs at $3100 \mathrm{~K}$, slightly later than for MD. For $\Delta / 2=0.14 R_{\text {eq }}$ as seen in Fig. 6(c), these temperatures are $1600 \mathrm{~K}, 600 \mathrm{~K}$, and $3000 \mathrm{~K}$, respectively. The crystallized structures obtained by cooling the liquid and heating the amorphous phase for $\Delta / 2=0.11 R_{\text {eq }}$ are both polycrystals shown at $303 \mathrm{~K}$ in Figs. 8(a) and 8(b). Of the two, the crystallized liquid has fewer defects, and its average atomic potential energy $E$ is closer to that in crystal. In fact, the crystallized liquid is a monocrystal containing just two small crystallites of different orientations. One is $4 \mathrm{~nm}^{3}$ and the other is $17 \mathrm{~nm}^{3}$ in the whole sample of $273.6 \mathrm{~nm}^{3}$. In contrast, the crystallized amorphous phase consists of many crystallites of different sizes. The size of the biggest is $74 \mathrm{~nm}^{3}$, which is $27 \%$ of the whole volume of the system. The recrystallized liquid has larger grains because it was formed at significantly higher temperature.

When $\Delta / 2=0.06 R_{\text {eq }}$ and $0.11 R_{\text {eq }}$ are compared, it is clear that a greater $\Delta / 2$ value is analogous to a slower heating or cooling rate: conditions are such that crystallization can take place. Apparently the crystallization is enabled by the formation and diffusion of defects, such as those earlier observed in the crystal (Sec. IV B). Because a greater $\Delta / 2$ was found to generate more defects, it can be concluded that these defects act as vehicles in the process of crystallization. All this 


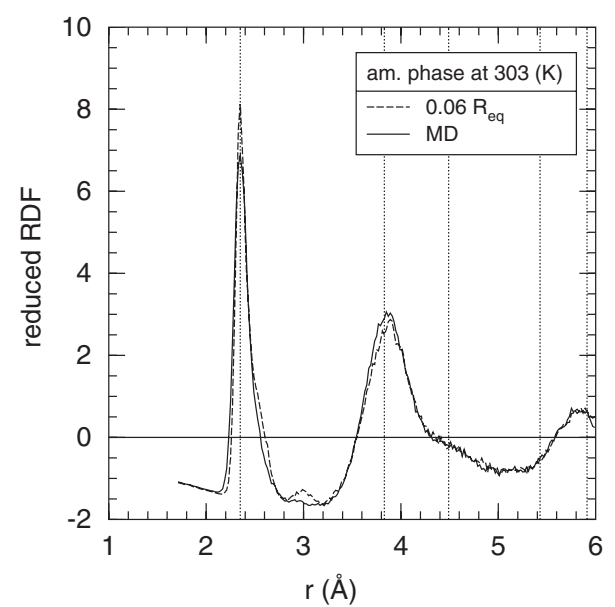

FIG. 8. Reduced RDFs for amorphous silicon at 303 (K) modeled by MD (Ref. 7) and by UFMC+ with $\Delta / 2=0.06 R_{\text {eq }}$.

has no general validity, however, because the even greater value $\Delta / 2=0.14 R_{\text {eq }}$ generates even more defects (Fig. 5), yet the crystallization behavior that was found [Fig. 6(c)] is rather unphysical. Compared to $\Delta / 2=0.11 R_{\text {eq }}[$ Fig. 6(b)], it is hard to understand why the liquid would crystallize at a lower temperature and the amorphous phase at a higher temperature. Apparently $\Delta / 2=0.14 R_{\mathrm{eq}}$ introduces spurious effects and is a too high value to study these phase transitions by UFMC.

Finally we will use the RDFs of Fig. 9 to have a look at the liquids produced by UFMC+ and compare them with the MD liquid (bottom curve). The two middle curves are the UFMC+ liquids formed with $\Delta / 2=0.06 R_{\text {eq }}$ and $\Delta / 2=0.11 R_{\text {eq }}$ just above the melting point. The results for $\Delta / 2=0.11 R_{\mathrm{eq}}$ are almost indistinguishable from the MD results, which is a very encouraging results. For $\Delta / 2=0.06 R_{\text {eq }}$, the maxima and minima of the RDF lie at the same positions but they are somewhat more pronounced. The average coordination number in this liquid is 5.33, which is slightly lower than the values 5.52 for UFMC+ with $\Delta / 2=0.11 R_{\text {eq }}$ and 5.65 for MD.

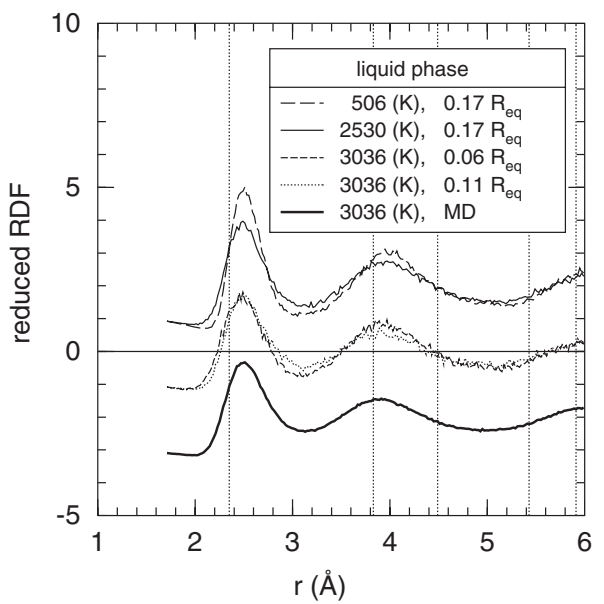

FIG. 9. Reduced RDFs and the liquid at 3036 (K) modeled by MD (Ref. 7, bottom curve), by UFMC+ with $\Delta / 2=0.06 R_{\text {eq }}$ and $\Delta / 2=0.1 R_{\text {eq }}$ (middle two curves), and by applying UFMC+ with $\Delta / 2=0.17 R_{\text {eq }}$ to the crystal at the two temperatures indicated.
Earlier it was shown that using the high value $\Delta / 2$ $=0.17 R_{\text {eq }}$ and apply UFMC + to the silicon crystal at temperatures below the melting temperature produces a system that stays in a disordered state. The two top curves in Fig. 9 show the results of this treatment for two temperatures, 506 and $2530 \mathrm{~K}$. It can be seen that in both cases, the RDFs are surprisingly similar to the MD liquid. Although the coordination numbers are a little different,5.35 at $506 \mathrm{~K}$ and 5.27 at $2530 \mathrm{~K}$ versus 5.65 for $\mathrm{MD},{ }^{7}$ it is safe to say that a too high value of $\Delta / 2$ lets UFMC+ produce a liquid under conditions where the crystalline phase is thermodynamically stable.

In conclusion, we find that UFMC+ models phase transitions and produces liquid and amorphous structures comparable with MD and in a smaller number of iteration steps. It can be applied very efficiently for crystallization and relaxation of the amorphous phase. The UFMC parameters have to be chosen according to the simulation of the desired process. Using the lower value $\Delta / 2=0.06 R_{\text {eq }}$ would be more appropriate for relaxation. Comparison of the $\mathrm{MD}$ and UFMC+ results shows that amorphization and glass transition take place at the same temperatures. To observe crystallization of the amorphous phase upon heating or even of the liquid upon cooling (something not possible with MD with cooling rates as low as $0.91 \mathrm{~K} / \mathrm{ps}$ ), higher values $\Delta / 2 \geq 0.11 R_{\text {eq }}$ have to be applied. The crystallized amorphous phase is a polycrystal.

\section{Annealing of the amorphous phase produced by sputter bombardment}

To see how UFMC performs in a more complicated situation, we have carried out UFMC ++ simulations of annealing a Si crystal with two (001) surfaces, one kept static and one left free. The free surface had been amorphized by prior $500 \mathrm{eV}$ Ar bombardment. ${ }^{6}$ The amorphous phase is approximately 25-30 $\AA$ thick and is characterized by a mean coordination number of 4.63 at zero temperature, a density varying within $5 \%$ of the crystal density, and a maximum local QV concentration of $18 \%$ (in the middle of the amorphous part) ${ }^{8}$ As a side remark, we mention that compared with the amorphous phase produced by cooling the liquid to room temperature (Sec. IV C), the amorphous phase produced by particle bombardment has a higher coordination number and virtually the same QV concentration. The difference in coordination number shows that by cooling the liquid, the formed amorphous phase is more relaxed. This seems reasonable because the temperature started from a value as high as 2990 $\mathrm{K}$ while during bombardment most of the time it was kept at $303 \mathrm{~K}$. The purpose of the annealing simulations was to study recrystallization by UFMC ++ . The difference with the crystallization of the amorphous phase discussed in Sec. IV C is that here the amorphous phase has a "natural" interface with the crystalline phase so that many nucleation sites for crystallization are present.

The UFMC annealing temperatures $T$ range from 303 to $2530 \mathrm{~K}$, all below the MEAM-L melting temperature of 2990 $\mathrm{K}$, and the values used for $\Delta / 2$ are $0.06 R_{\mathrm{eq}}, 0.11 R_{\mathrm{eq}}$, and $0.14 R_{\text {eq }}$. The outcome of the simulations is shown in Figs. 10 and 11 , where the UFMC++ results are compared with the 

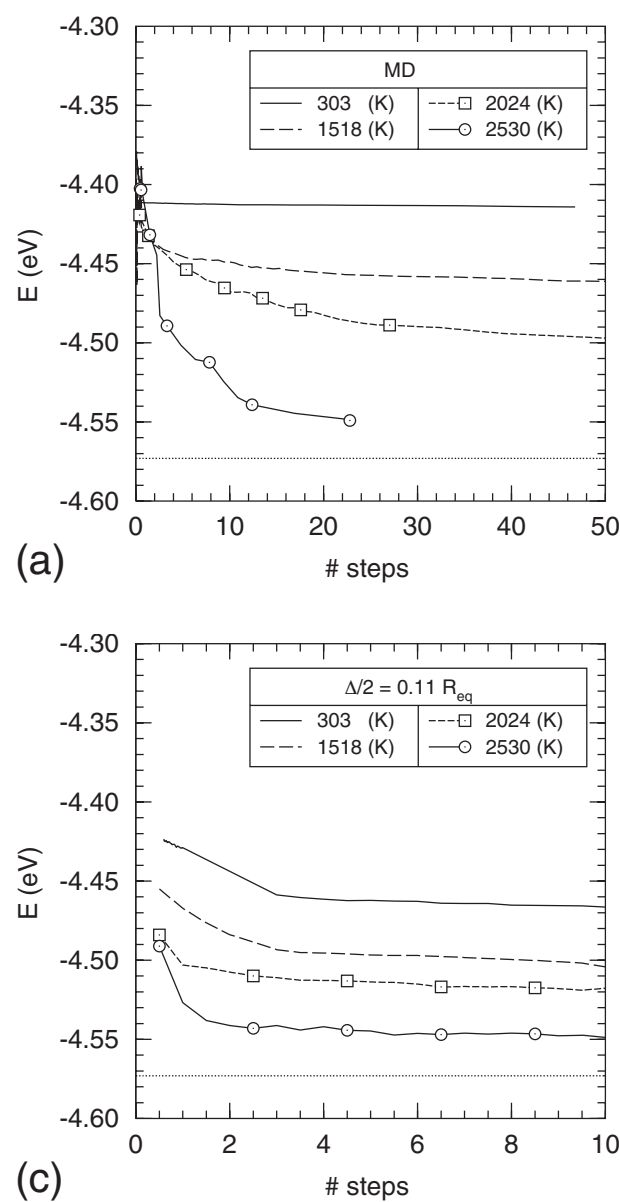

(b)
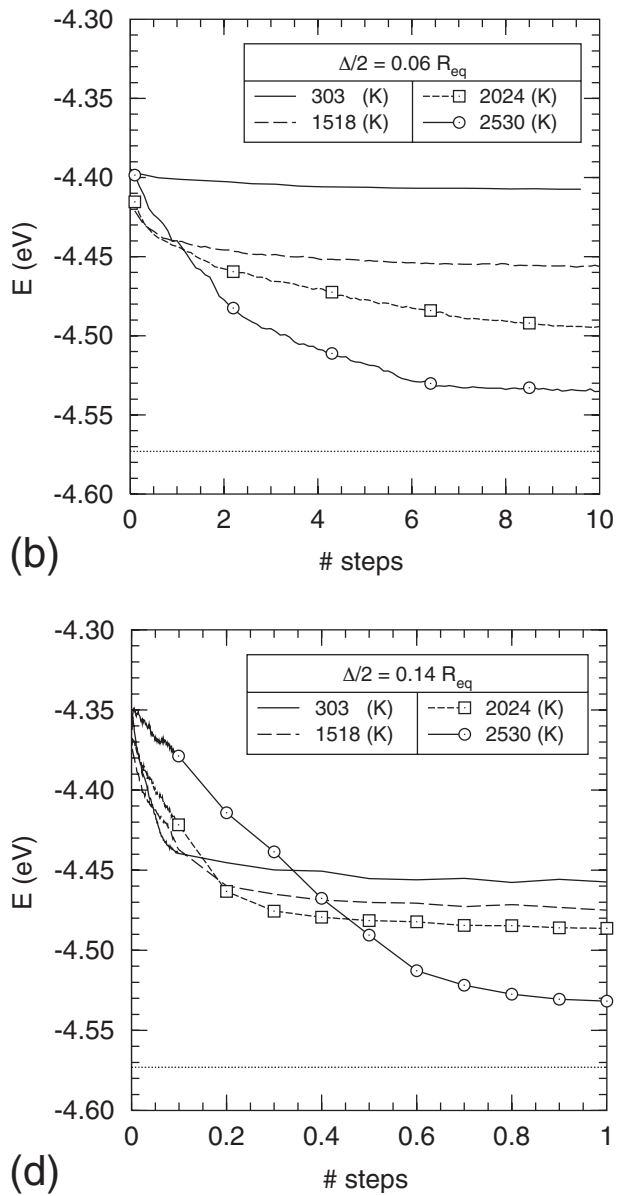

FIG. 10. Average potential energy $E$ per atom versus number of iteration steps divided by $10^{5}$ in (a) MD simulations (Ref. 9) and in [(b)-(d)] UFMC ++ simulations with $\Delta / 2=0.06 R_{\text {eq }}, 0.11 R_{\text {eq }}$, and $0.14 R_{\text {eq }}$, respectively. The four different annealing temperatures are indicated in the legends. The horizontal dashed line in each figure denotes the energy of a perfect crystal with a dimerized (100) surface.
MD results, ${ }^{8}$ which were obtained at the same temperatures followed by quenching the system to $0 \mathrm{~K}$. Figure 10 shows the average potential energy $E$ per atom versus the number of iteration steps in the simulations. Figure 11 shows snapshots of the system at the end of these runs and lists the percentage of evaporated Ar atoms and the maximum local QV concentration at the end. As a special reproducibility test, we have repeated some of the UFMC ++ simulations under the same conditions and with the same initial configurations. The potential-energy curves reproduced very closely, showing that in spite of the random moves of the atoms, the UFMC method converges to stable results.

It is found that after the UFMC ++ simulations with $\Delta / 2=0.06 R_{\mathrm{eq}}$ at all temperatures, the recrystallized amorphous-phase fraction is about the same as after MD (Fig. 11). In addition, the final potential energies are also nearly the same [Figs. 10(a) and 10(b)] but number of steps needed in UFMC ++ to reach the same level as in MD is only about $20 \%$ of that in MD. On this accelerated scale, $\mathrm{UFMC}++$ lags slightly behind MD in removing the quasivacancies and in letting the Ar impurity atoms evaporate. At $303 \mathrm{~K}$, there is no recrystallization but only a relaxation of the amorphous phase. The average coordination number decreases from 4.63 to 4.61 , the potential energy drops only slightly (0.01 eV/atom), and the concentration of QVs stays the same. Partial recrystallization is observed at $1518 \mathrm{~K}$, and the temperature has to be increased to $T=2530 \mathrm{~K}$ before (almost) complete recrystallization of the amorphous phase occurs. The potential energy then decreases by 0.14 to $-4.54 \mathrm{eV} /$ atom, a value only $0.04 \mathrm{eV}$ higher than that of the perfect crystal with two surfaces. This difference is due to the fact that there are still impurities (Ar atoms, which have a very high potential energy compared with $\mathrm{Si}$ atoms) and other defects in the recrystallized amorphous phase.

For $\Delta / 2 \geq 0.11 R_{\text {eq }}$, the amorphous phase fully recrystallizes already at $303 \mathrm{~K}$ (Fig. 11), although the surface in the $303 \mathrm{~K}$ picture of Fig. 11 for $\Delta / 2=0.11 R_{\text {eq }}$ seems to suggest otherwise (we come back to this). In spite of this full recrystallization, the potential-energy curves in Figs. 10(c) and 10 (d) do not all decrease to $-4.54 \mathrm{eV} /$ atom, the value just found for full crystallization. The reason lies in the unequal balance of positive and negative contributions to the energy. The residual defects have a stronger effect on the energy than the crystallization of the amorphous phase. Coming back to the surface region of the system annealed at $303 \mathrm{~K}$ with $0.11 R_{\text {eq }}$, which does not look recrystallized, one sees on closer inspection that it in fact contains two crystallites of different orientation than the whole crystal. These crystallites, a few monolayers thick, were formed because of accumulation of the defects that were left behind after the passing of the crystallization front. Later these defects reorganized and isolated two small crystallite parts from the rest of the crystal. Apparently the presence of these defects made it energetically favorable for the crystallites to appear in a different orientation.

For the UFMC ++ simulations, the general trends observed are that increasing $T$ and increasing $\Delta / 2$ decrease the 


\begin{tabular}{|c|c|c|c|c|}
\hline & \multirow[t]{2}{*}{ MD } & \multicolumn{3}{|c|}{ UFMC++ } \\
\hline & & $\Delta / 2=0.06 R_{\mathrm{eq}}$ & $\Delta / 2=0.11 R_{\mathrm{eq}}$ & $\Delta / 2=0.14 R_{\mathrm{eq}}$ \\
\hline & Whn & & סדיר & 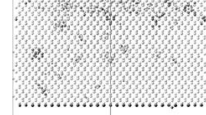 \\
\hline 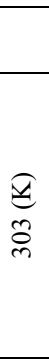 & $\begin{array}{c}0 \% \text { Ar evapor. } \\
17 \% \text { QV }\end{array}$ & $\begin{array}{c}0 \% \text { Ar evapor. } \\
17 \% \text { QV }\end{array}$ & $\begin{array}{c}2.5 \% \mathrm{Ar} \text { evapor. } \\
2.5 \% \mathrm{QV}\end{array}$ & $\begin{array}{c}17 \% \text { Ar evapor. } \\
2.5 \% \mathrm{QV}\end{array}$ \\
\hline \multirow[t]{2}{*}{$\begin{array}{l}2 \\
\infty \\
\infty\end{array}$} & 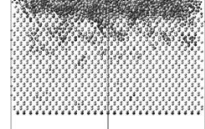 & 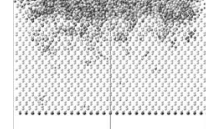 & 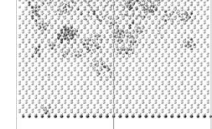 & \\
\hline & $\begin{array}{c}8.7 \% \text { Ar evapor. } \\
16 \% \mathrm{QV}\end{array}$ & $\begin{array}{c}3.6 \% \mathrm{Ar} \text { evapor. } \\
11 \% \mathrm{QV}\end{array}$ & $\begin{array}{c}33 \% \text { Ar evapor. } \\
2 \% \text { QV }\end{array}$ & $\begin{array}{c}15 \% \text { Ar evapor. } \\
4 \% \text { QV }\end{array}$ \\
\hline \multirow[t]{2}{*}{ 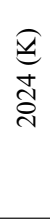 } & 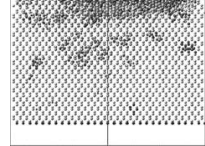 & W & 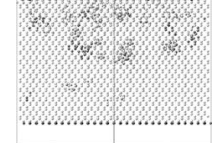 & 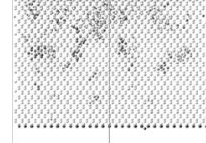 \\
\hline & $\begin{array}{c}12 \% \text { Ar evapor. } \\
8 \% \text { QV }\end{array}$ & $\begin{array}{c}8,7 \% \text { Ar evapor. } \\
8 \% \mathrm{QV}\end{array}$ & $\begin{array}{c}71 \% \text { Ar evapor. } \\
1.8 \% \mathrm{QV}\end{array}$ & $\begin{array}{c}19 \% \text { Ar evapor. } \\
2.5 \% \mathrm{QV}\end{array}$ \\
\hline \multirow[t]{2}{*}{$\begin{array}{l}\sqrt{2} \\
0 \\
\tilde{n} \\
\end{array}$} & 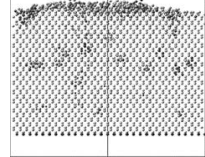 & 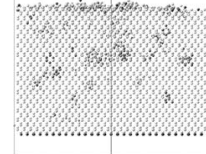 & 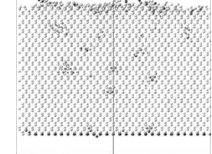 & 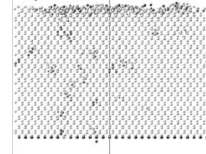 \\
\hline & $\begin{array}{c}88 \% \text { Ar evapor. } \\
2 \% \text { QV }\end{array}$ & $\begin{array}{c}60 \% \text { Ar evapor. } \\
1 \% \mathrm{QV}\end{array}$ & $\begin{array}{c}78 \% \text { Ar evapor. } \\
1 \% \mathrm{QV}\end{array}$ & $\begin{array}{c}89 \% \text { Ar evapor. } \\
1 \% \text { QV }\end{array}$ \\
\hline
\end{tabular}

FIG. 11. Views of the systems after annealing at different temperatures by MD (Ref. 9) and by UFMC ++ with $\Delta / 2=0.06 R_{\text {eq }}, 0.11 R_{\text {eq }}$, and $0.14 R_{\text {eq. }}$. The percentage of evaporated Ar atoms and the maximum local QV concentration at the end are indicated for each case.

number of residual defects (Fig. 11). However, there are exceptions. For $\Delta / 2=0.14 R_{\text {eq }}$, the system still contains unexpectedly high concentrations of residual defects after $\mathrm{UFMC}++$ at 2024 and $1518 \mathrm{~K}$. At $303 \mathrm{~K}$, the situation is less clear but especially the QV concentration is still quite large. All this might be connected with the crystallization speed. Contrary to the situation for lower values of $\Delta / 2$, for $0.14 R_{\mathrm{eq}}$ the recrystallization proceeds faster at lower temperatures [Fig. 11(d)]. This effect is very likely related to the steep increase in the quasivacancy concentration with decreasing temperature for $0.14 R_{\mathrm{eq}}$ (Fig. 5). When the crystallization front passes fast, Ar atoms, which are concentrated in the amorphous phase, do not have enough time to reach the surface and evaporate. As a result, they stay in the crystal. Figure $10(\mathrm{~d})$ shows that up to $2024 \mathrm{~K}$, crystallization is completed within $0.5 \times 10^{5}$, which is apparently too soon. At the highest temperature $(2530 \mathrm{~K})$, the crystallization speed is slower so more Ar atoms evaporate. With $\Delta / 2 \leq 0.11 R_{\text {eq }}$, the crystallization speed is slow enough at all $T$.

Another unexpected observation with $0.14 R_{\mathrm{eq}}$ is the substantial increase in the potential energy shortly after starting the UFMC ++ simulation. This increase becomes clear if Fig. 10(d) is compared with Fig. 10(b). One would expect that the final MD part of UFMC ++ would bring the system after UFMC back to its "proper" energy value, just as was observed earlier for crystalline $\mathrm{Si}$. This is not what happens. Apparently the presence of a sizeable amorphous phase slows down or obstructs the removal of the many defects generated with $0.14 R_{\mathrm{eq}}$ by the $1.5 \mathrm{ps} \mathrm{MD}$ runs. As soon as most of this phase is recrystallized, however, the MD runs following UFMC are capable to return the energy to its proper value.

Looking at the process dynamics as a whole, for all investigated values of $\Delta / 2$ and $T$, recrystallization in UFMC proceeds in the same way as in MD. ${ }^{8}$ It starts at the amorphous-crystal interface and advances roughly logarithmically with the number of iteration steps (Fig. 10). This nonlinear behavior is very likely due to the proximity of the surface, which progressively slows down the dynamics as the crystalline/amorphous interface approaches. During recrystallization, $\mathrm{Si}$ and $\mathrm{Ar}$ atoms are highly mobile. They diffuse over distances in the ranges $9-14 \AA(\mathrm{Si})$ and 25-30 $\AA$ (Ar), depending on $T$, and the atoms completely change their local neighbors. The observed diffusion is comparable with MD. Defects that were in the crystal near the interface before annealing annihilate only partly. Defects that were in the amorphous phase and stayed in the system after the passing of the recrystallization front diffuse and randomly annihilate. If defects reach the surface, they evaporate. 
From all evidence presented it can be concluded that $\mathrm{UFMC}++$ performs well in modeling recrystallization of a partly amorphized silicon crystal during annealing. The recrystallization proceeds in a very similar way as in MD but needs fewer iteration steps. The value $0.11 R_{\text {eq }}$ for $\Delta / 2$ seems to be a good compromise between physically meaningful results and computational efficiency. When the process itself is less important and one needs to crystallize a system in an even faster way, a value of $0.14 R_{\text {eq }}$ or possibly even higher can be recommended. With $\Delta / 2=0.06 R_{\text {eq }}$, UFMC can be applied for relaxation of the amorphous phase. As an example, it can be used in simulations of surface bombardment to speed up the relaxation of the formed amorphous phase, and thereby to artificially increase the time between successive projectile impacts in order to let the projectile flux appear to be closer to the experimental value.

\section{CONCLUSIONS}

We have studied the applicability of the UFMC method, determined safe parameter limits, within which UFMC simulations produce results comparable with MD, and identified the main ways in which UFMC operates. It has been found that when the parameter values are within their limits, the main asset of the UFMC method is the computational speed increase in UFMC over MD, which can be very high and mostly depends on the UFMC parameter $\Delta / 2$. At the same time, the UFMC method followed by a short MD equilibration approximates real process dynamics and gives proper physical properties of the system.

We demonstrated that the UFMC method can be applied to simulations of a large variety of processes: surface diffusion, amorphization, melting, glass transition, and crystallization. However, if the UFMC parameters are too high, the method can give unphysical results. In this respect, the choice of $\Delta / 2$ is particularly critical. To perform reliable simulations, proper values of the two UFMC parameters $\Delta / 2$ and $T$ should be chosen. A reasonable criterion is that a perfect crystal after UFMC+ or UFMC++ (i.e., UFMC followed by a short MD equilibration) should still be a crystal, without the formation of additional defects and-if this is important—with all atoms at their original positions. This effectively says that processes with a lower activation energy than vacancy-interstitial formation are allowed to happen. This criterion is satisfied for crystalline $\mathrm{Si}$ with the MEAM-L potential by $\Delta / 2 \leq 0.15 R_{\text {eq }}$ and $T$ about or above room temperature, i.e., when the average potential energy stays below -3.5 eV during UFMC.

For simulation of phase transitions, performed as a test of UFMC and presented as cases where UFMC prevails over $\mathrm{MD}$, the choice of the UFMC parameter values depends on the desired computational speed and on either the concentration of residual defects or on the degree of relaxation of the system. To be on a safe side, we would recommend the following values for silicon: (1) for amorphization of the liquid and the glass transition $-\Delta / 2=0.06 R_{\text {eq }}$ and $T$ about $2000 \mathrm{~K}$ and $2100 \mathrm{~K}$, respectively; (2) for melting of the crystal, with nucleation centers $-\Delta / 2 \leq 0.14 R_{\text {eq }}$ and $T$ about $2950 \mathrm{~K}$, the MD melting temperature; (3) for crystallization of the liquid $-\Delta / 2=0.11 R_{\text {eq }}$ and $T \leq 2000 \mathrm{~K}$; (4) for crystallization of a homogeneous amorphous phase $-\Delta / 2=0.11 R_{\text {eq }}$ and $T \geq 300 \mathrm{~K}$; and (5) for crystallization of an amorphous phase with nucleation point-either $\Delta / 2=0.06 R_{\mathrm{eq}}$ and $T \geq 2024 \mathrm{~K}$ or $\Delta / 2=0.11 R_{\mathrm{eq}}$ and any $T$.

The crystallized liquid and homogeneous amorphous phase are polycrystals. At higher annealing temperatures, the size of the grains is bigger and their number is smaller. The crystallized heterogeneous amorphous phase is a single crystal with concentration of residual defects depending on $T$. At higher $T$, this concentration is smaller and the computational speed is higher. Structural properties of the amorphous phase and liquid are similar to ones in MD simulation. Therefore, UFMC and MD methods can be effectively applied in turns, if needed. All that is needed is just a few extra program lines in an MD code. As an example of the attainable acceleration, the number of iteration steps needed to get the same relaxed amorphous phase during cooling the liquid is at least two orders of magnitude smaller in UFMC than in MD.

In all the simulations, the UFMC parameter $T$ should have a physically meaningful value. With small $\Delta / 2\left(0.06 R_{\mathrm{eq}}\right)$, results are very similar to ones in $\mathrm{MD}$, and therefore it can be considered as a real temperature. However, for higher $\Delta / 2$, it is a parameter rather than a real temperature and should be treated carefully.

The main microscopic factor that allows crystallization of the amorphous phase at low $T$ and even crystallization of the liquid is the large displacements of atoms compared to MD. Because of these large displacements of all the atoms, interatomic spaces (quasivacancies) are continuously created and annihilated and in some cases, atoms exchange their positions. The creation of interatomic spaces is accompanied by a large increase in the average atomic potential energy. This effect is opposite to MD and it causes the conditions for crystallization to be more favorable than in MD.

Finally, the present work almost exclusively applies to $\mathrm{Si}$. For other materials, the safety limits of the UFMC parameters may be somewhat different. Therefore, to prevent artifacts, new calibrations should be performed for new materials. This work may be used as a guideline for how to do this.

\section{ACKNOWLEDGMENTS}

This research was carried out under the Project No. 02EMM32 in the framework of the Research Program of the Materials innovation institute M2i (www.m2i.nl), the former Netherlands Institute for Metals Research, and the Stichting voor Fundamenteel Onderzoek der Materie [FOM, financially supported by the Nederlandse Organisatie voor Wetenschappelijk Onderzoek (NWO)]. 
*Corresponding author. FAX: +31 $15 \quad 278$ 6730; m.timonova@tudelft.nl

${ }^{1}$ M. Rao, C. Pangali, and B. J. Berne, Mol. Phys. 37, 1773 (1979).

${ }^{2}$ S. Goldman, J. Comput. Phys. 62, 441 (1986); S. Singh and S. Goldman, ibid. 62, 464 (1986).

${ }^{3}$ G. Dereli, Mol. Simul. 8, 351 (1992).

${ }^{4}$ C. H. Grein, R. Benedek, and T. de la Rubia, Comput. Mater. Sci. 6, 123 (1996).

${ }^{5}$ D. J. Oh and R. A. Johnson, J. Mater. Res. 3, 471 (1988).
${ }^{6}$ M. Timonova, B.-J. Lee, and B. J. Thijsse, Nucl. Instrum. Methods Phys. Res. B 255, 195 (2007).

${ }^{7}$ M. Timonova and B. J. Thijsse, Comput. Mater. Sci. (in print). ${ }^{8}$ M. Timonova and B. J. Thijsse (unpublished).

${ }^{9}$ W. Eckstein, Computer Simulation of Ion-Solid Interactions (Springer, Heidelberg, 1991), p. 40.

${ }^{10}$ A. F. Voter, F. Montalenti, and T. C. Germann, Annu. Rev. Mater. Res. 32, 321 (2002).

${ }^{11}$ J. B. Adams, Z. Wang, and Y. Li, Thin Solid Films 365, 201 (2000). 